EPJ Web of Conferences 78, 02005 (2014)

DOI: $10.1051 /$ epjconf / 20147802005

(C) Owned by the authors, published by EDP Sciences, 2014

\title{
Quantumness of discrete Hamiltonian cellular automata
}

\author{
Hans-Thomas Elze ${ }^{a}$ \\ Dipartimento di Fisica "Enrico Fermi", Università di Pisa, Largo Pontecorvo 3, I-56127 Pisa, Italia
}

\begin{abstract}
We summarize a recent study of discrete (integer-valued) Hamiltonian cellular automata (CA) showing that their dynamics can only be consistently defined, if it is linear in the same sense as unitary evolution described by the Schrödinger equation. This allows to construct an invertible map between such CA and continuous quantum mechanical models, which incorporate a fundamental scale. Presently, we emphasize general aspects of these findings, the construction of admissible CA observables, and the existence of solutions of the modified dispersion relation for stationary states.
\end{abstract}

\section{Introduction}

The Cellular Automaton Interpretation of quantum mechanics has recently been laid out by G. 't Hooft [1]. The hope reflected in this far-reaching article, and in related works by others, is founded on the observation of quantum mechanical features arising in a large variety of deterministic "mechanical" models. While most of these models have been singular cases, i.e., which cannot easily be generalized to cover a realistic range of phenomena incorporating interactions, CA promise to provide the necessary versatility $[2,3]$.

The linearity of quantum mechanics $(\mathrm{QM})$ is a fundamental feature most notably embodied in the Schrödinger equation. This linearity does not depend on the particular object under study, provided it is sufficiently isolated from anything else. It is naturally reflected in the superposition principle and entails the "quantum essentials" interference and entanglement.

The linearity of QM has been questioned repeatedly and nonlinear modifications have been proposed, in order to test experimentally the robustness of QM against such nonlinear deformations. This has been thoroughly discussed by T.F. Jordan presenting a stepwise proof 'from within' QM that the theory has to be linear, given the additional separability assumption " ... that the system we are considering can be described as part of a larger system without interaction with the rest of the larger system." [4]

Recently, we have considered a seemingly unrelated discrete dynamical theory, which appears to deviate drastically from quantum theory, at first sight. However, we have shown that the deterministic mechanics of the class of Hamiltonian CA can be related to QM in the presence of a fundamental time scale. This relation demonstrates that consistency of the action principle of the underlying discrete dynamics implies, in particular, the linearity of both theories. This approach may offer additional insight into interference, entanglement, and measurement processes in QM, in the limit when the discreteness scale is negligible.

a e-mail: elze@df.unipi.it 


\section{CA Action Principle and observables}

The state of a classical cellular automaton (CA) with a denumerable set of degrees of freedom will be described by integer-valued "coordinates" $x_{n}^{\alpha}, \tau_{n}$ and "conjugated momenta" $p_{n}^{\alpha}, \pi_{n}$, where $\alpha \in \mathbf{N}_{0}$ denote different degrees of freedom and $n \in \mathbf{Z}$ different states. - The $x_{n}$ and $p_{n}$ might be higher dimensional vectors, while $\tau_{n}$ and $\mathcal{P}_{n}$ are assumed one-dimensional. We separate the "coordinate" $\tau_{n}$ from the $x_{n}^{\alpha}$ 's (correspondingly $\pi_{n}$ from the $p_{n}^{\alpha}$, s), since this degree of freedom represents the dynamical time variable here, discussed in $[2,3]$, see also further references there.

Finite differences, for all dynamical variables, are defined by:

$$
\Delta f_{n}:=f_{n}-f_{n-1} .
$$

Furthermore, we define (with summation convention for Greek indices, $r^{\alpha} s^{\alpha} \equiv \sum_{\alpha} r^{\alpha} s^{\alpha}$ ) $\mathcal{A}_{n}:=$ $\Delta \tau_{n}\left(H_{n}+H_{n-1}\right)+a_{n}, H_{n}:=\frac{1}{2} S_{\alpha \beta}\left(p_{n}^{\alpha} p_{n}^{\beta}+x_{n}^{\alpha} x_{n}^{\beta}\right)+A_{\alpha \beta} p_{n}^{\alpha} x_{n}^{\beta}+R_{n}, a_{n}:=c_{n} \pi_{n}$, where constants, $c_{n}$, and symmetric, $\hat{S} \equiv\left\{S_{\alpha \beta}\right\}$, and antisymmetric, $\hat{A} \equiv\left\{A_{\alpha \beta}\right\}$, matrices are all integer-valued; $R_{n}$ stands for higher than second powers in $x_{n}^{\alpha}$ or $p_{n}^{\alpha}$. The last definition determines the behaviour of the variable $\tau_{n}$; a very simple choice suffices here, cf. below.

Given these definitions, we introduce the integer-valued CA action:

$$
\mathcal{S}:=\sum_{n}\left[\left(p_{n}^{\alpha}+p_{n-1}^{\alpha}\right) \Delta x_{n}^{\alpha}+\left(\pi_{n}+\pi_{n-1}\right) \Delta \tau_{n}-\mathcal{A}_{n}\right]
$$

For an alternative but equivalent form, which is particularly suited for the discussion of symmetry properties, see Ref. [3]. - Furthermore, let integer-valued variations $\delta f_{n}$ be applied to a polynomial $g$ in this way:

$$
\delta_{f_{n}} g\left(f_{n}\right):=\left[g\left(f_{n}+\delta f_{n}\right)-g\left(f_{n}-\delta f_{n}\right)\right] / 2 \delta f_{n},
$$

and $\delta_{f_{n}} g \equiv 0$, if $\delta f_{n}=0$. - Then, CA dynamics is introduced by the following postulate.

Action Principle. The discrete evolution of a CA is determined by the stationarity of its action under

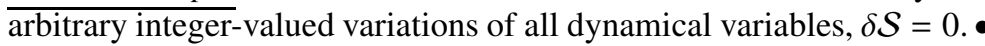

Several features of this Action Principle are worth emphasizing:

i) Variations of terms that are constant, linear, or quadratic (in dynamical variables) yield analogous results as infinitesimal variations of corresponding real-valued terms.

ii) While infinitesimal variations do not conform with integer valuedness, there is a priori no restriction of integer variations, hence arbitrary integer-valued variations must be admitted.

iii) However, for arbitrary variations $\delta f_{n}$, the remainder of higher powers $R_{n}$ in $H_{n}$, which ultimately enters the action, has to vanish for consistency. Otherwise the number of equations of motion generated by variation of the action, generally, would exceed the number of variables. (However, a suitably chosen $R_{0}$ or a sufficient small number of such remainder terms can serve to encode the initial conditions for the CA evolution.)

Employing the notation $\dot{O}_{n}:=O_{n+1}-O_{n-1}$, discrete analogues of Hamilton's equations are obtained by variation of the CA action $\mathcal{S}$ (keeping $R_{n} \equiv 0$ ):

$$
\begin{gathered}
\dot{x}_{n}^{\alpha}=\dot{\tau}_{n}\left(S_{\alpha \beta} p_{n}^{\beta}+A_{\alpha \beta} x_{n}^{\beta}\right), \quad \dot{p}_{n}^{\alpha}=-\dot{\tau}_{n}\left(S_{\alpha \beta} x_{n}^{\beta}-A_{\alpha \beta} p_{n}^{\beta}\right), \\
\dot{\tau}_{n}=c_{n}, \quad \dot{\pi}_{n}=\dot{H}_{n},
\end{gathered}
$$

where all terms are integer-valued. Discreteness of the automaton time $n$ is reflected by finite difference equations here. Their appearance has motivated the name Hamiltonian CA. 
Further aspects of these equations, in particular the ensuing symmetries and conservation laws, have been discussed in Refs. [2, 3]. - The equations are time reversal invariant. Most remarkably, they give rise to conservation laws that are in one-to-one correspondence with those of the Schrödinger equation for the Hamilton operator $\hat{H}$ given through the integer-valued symmetric and antisymmetric matrices (cf. above), $\hat{S} \equiv\left\{S_{\alpha \beta}\right\}$ and $\hat{A} \equiv\left\{A_{\alpha \beta}\right\}$, respectively: $\hat{H}:=\hat{S}+i \hat{A}$. - These observations are based on the fact that the Eqs. (4) can be combined into:

$$
\dot{x}_{n}^{\alpha}+i \dot{p}_{n}^{\alpha}=-i \dot{\tau}_{n} H_{\alpha \beta}\left(x_{n}^{\beta}+i p_{n}^{\beta}\right),
$$

and its adjoint, employing the matrix elements of $\hat{H}$. This presents the discrete analogue of Schrödinger's equation, with $\psi_{n}^{\alpha}:=x_{n}^{\alpha}+i p_{n}^{\alpha}$ as the amplitude of the " $\alpha$-component" of "state vector" $|\psi\rangle$ at "time" $n$

Presently, we would like to draw attention to another surprising parallel between the discrete and continuum models, CA and quantum mechanics, respectively. - We may try to define a "Poisson bracket" related to the dynamical variables of the CA, which are denoted collectively by $X_{n}, P_{n}$ and which represent the $x_{n}^{\alpha}, \tau_{n}$ and $p_{n}^{\alpha}, \pi_{n}$, respectively:

$$
\{A, B\}:=\sum_{n}\left(\delta_{X_{n}} A \delta_{P_{n}} B-\delta_{X_{n}} B \delta_{P_{n}} A\right),
$$

employing the variational derivative defined in Eq. (3), since ordinary derivatives are not available; here $A$ and $B$ are polynomials depending on the dynamical variables.

However, inspection shows that such polynomials $A$ and $B$ cannot be arbitrarily chosen, in order to have a consistent bracket which, besides showing bilinearity and antisymmetry, also leads to derivation-like product formula and Jacobi identity, the defining properties of a Lie bracket operation. - Namely, the problem arises that generally the result of the bracket operation might depend on the integer-valued variations $\delta f_{n}$, which enter through the definition of the variational derivative, Eq. (3). This would prohibit to form a closed algebra of polynomials. However, recalling observation i) above, we restrict the polyomials to be constant, linear, or quadratic (in dynamical variables). They form a closed algebra with respect to the bracket operation, which becomes consistent in all respects.

This simple result is remarkable for two reasons. - First, the Hamilton operator $\hat{H}$ defines a quadratic form in terms of the $x_{n}^{\alpha}$ and $p_{n}^{\alpha}$, which can be compactly written as $\mathcal{H}:=\sum_{n} \psi_{n}^{* \alpha} H_{\alpha \beta} \psi_{n}^{\beta} / 2$. It corresponds to the expectation $\langle\psi|\hat{H}| \psi\rangle$ in quantum mechanics written in the particular representation developed by A. Heslot [5]. This expectation belongs to the observables of a quantum mechanical object and should belong to the CA observables as well. In particular, since Eq. (6) can be rewritten as $\dot{\psi}_{n}^{\alpha}=\dot{\tau}_{n}\left\{\psi_{n}^{\alpha}, \mathcal{H}\right\}$. - Second, restricting ourselves to quadratic forms in the dynamical variables as CA observables (eliminating trivial constant and linear forms that would yield inhomogeneous evolution equations), we arrive at a closed algebra of observables with respect to the Poisson bracket operation (7).

We recall that all quantum mechanical observables are generated by Hermitean operators in this way as quadratic forms [5]. - Thus, insisting on the Hamiltonian structure of CA dynamics, including a suitably defined Poisson bracket, we are able to extend the close correspondence between CA and quantum mechanical systems to include the structure of the observables as well.

\section{$3 \mathrm{CA} \leftrightarrow \mathrm{QM}$ map and modified dispersion relation}

The correspondence that we discussed is not accidental and can be understood with the help of an invertible map between Hamiltonian CA and quantum mechanical objects that are characterized by 
a fundamental discreteness scale $l$. Implications for the conservation laws on both sides of the map were described in Refs. [2, 3]. Here we reconsider the resulting dispersion relation, which might have observable consequences.

We employ Shannon's Sampling Theorem [6]: Consider square integrable bandlimited functions $f$, i.e., which can be represented as $f(t)=(2 \pi)^{-1} \int_{-\omega_{\max }}^{\omega_{\max }} \mathrm{d} \omega \mathrm{e}^{-i \omega t} \tilde{f}(\omega)$, with bandwidth $\omega_{\max }$. Given the set of amplitudes $\left\{f\left(t_{n}\right)\right\}$ for the set $\left\{t_{n}\right\}$ of equidistantly spaced times (spacing $\pi / \omega_{\max }$ ), the function $f$ is obtained for all $t$ by:

$$
f(t)=\sum_{n} f\left(t_{n}\right) \frac{\sin \left[\omega_{\max }\left(t-t_{n}\right)\right]}{\omega_{\max }\left(t-t_{n}\right)} .
$$

Since the CA "time" is given by an integer $n$, the discrete physical time is obtained by multiplying with the scale $l, t_{n} \equiv n l$, and the bandwidth by $\omega_{\max }=\pi / l$. - Next, we insert $\psi_{n}^{\alpha}:=x_{n}^{\alpha}+i p_{n}^{\alpha}$ in Eq. (6) and apply the Sampling Theorem, which maps this discrete time equation invertibly to a continuous time equation:

$$
2 \sinh \left(l \partial_{t}\right) \psi^{\alpha}(t)=\frac{1}{i} H_{\alpha \beta} \psi^{\beta}(t)
$$

incorporating the simplest choice $\dot{\tau}_{n} \equiv 1$. This is recognized as the Schrödinger equation, however, modified in important ways. - The wave function $\psi^{\alpha}$ now is bandlimited by $\omega_{\text {max }}$, which amounts to an ultraviolet cut-off of the energy $E$ of stationary states, $\psi_{E}(t):=\exp (-i E t) \tilde{\psi}$. Diagonalizing the self-adjoint Hamiltonian, $\hat{H} \rightarrow \operatorname{diag}\left(\epsilon_{0}, \epsilon_{1}, \ldots\right)$, Eq. (9) yields the eigenvalue equation, $\sin \left(E_{\alpha} l\right)=$ $\epsilon_{\alpha} / 2=: \bar{\epsilon}_{\alpha}$, or, $E_{\alpha}=l^{-1} \arcsin \left(\bar{\epsilon}_{\alpha}\right)=l^{-1} \bar{\epsilon}_{\alpha}\left[1+\bar{\epsilon}_{\alpha}^{2} / 3 !+\mathrm{O}\left(\bar{\epsilon}_{\alpha}^{4}\right)\right]$. Thus, we obtain a modified dispersion relation.

Most importantly, discrete Hamiltonians do indeed exist which have their spectrum bounded between -2 and 2, such that our eigenvalue equation has real solutions. A complete classification of such integer-valued symmetric matrices has recently been given [7]. This is a subject for future extension and physical interpretation, while all problems related to measurements in QM and their correlates in the Hamiltonian CA picture have still been left untouched.

Acknowledgements: I thank G. 't Hooft and L. Maccone for discussions and correspondence, P. Ádám, T. Bíró, P. Lévai and S. Varró for inviting me to the inspiring "Wigner 111 - Colourful \& Deep" symposium (Budapest, November 2013), and L. Diósi for discussions and kind hospitality during this conference.

\section{References}

[1] G. 't Hooft, preprint arXiv:1405.1548 (2014)

[2] H.-T. Elze, Phys. Rev. A 89, 012111 (2014) [arXiv:1312.1615]

[3] H.-T. Elze, J. Phys.: Conf. Ser. 504, 012004 (2014)

[4] T.F. Jordan, Phys. Rev. A 73, 022101 (2006); do. J. Phys.: Conf. Ser. 196, 012010 (2009)

[5] A. Heslot, Phys. Rev. D 31, 1341 (1985)

[6] A.J. Jerri, Proc. IEEE 65, 1565 (1977)

[7] J.F. McKee and C.J. Smyth, J. Algebra 317, no. 1, 260-290 (2007) 